\title{
Isotropic inelastic and superelastic collisional rates in a multiterm atom
}

\author{
L. Belluzzi ${ }^{1,2}$, E. Landi Degl'Innocenti ${ }^{3}$, and J. Trujillo Bueno ${ }^{1,2,4}$ \\ ${ }^{1}$ Instituto de Astrofísica de Canarias, C. vía Láctea s/n, 38205 La Laguna, Tenerife, Spain \\ e-mail: belluzzi@iac.es \\ 2 Departamento de Astrofísica, Facultad de Física, Universidad de La Laguna, 38206 La Laguna, Tenerife, Spain \\ 3 Dipartimento di Fisica e Astronomia, Università di Firenze, Largo E. Fermi 2, 50125 Firenze, Italy \\ ${ }^{4}$ Consejo Superior de Investigaciones Científicas, Spain
}

Received 6 October 2012 / Accepted 4 November 2012

\begin{abstract}
The spectral line polarization of the radiation emerging from a magnetized astrophysical plasma depends on the state of the atoms within the medium, whose determination requires considering the interactions between the atoms and the magnetic field, between the atoms and photons (radiative transitions), and between the atoms and other material particles (collisional transitions). In applications within the framework of the multiterm model atom (which accounts for quantum interference between magnetic sublevels pertaining either to the same $J$-level or to different $J$-levels within the same term) collisional processes are generally neglected when solving the master equation for the atomic density matrix. This is partly due to the lack of experimental data and/or of approximate theoretical expressions for calculating the collisional transfer and relaxation rates (in particular the rates for interference between sublevels pertaining to different $J$-levels, and the depolarizing rates due to elastic collisions). In this paper we formally define and investigate the transfer and relaxation rates due to isotropic inelastic and superelastic collisions that enter the statistical equilibrium equations for the atomic density matrix of a multiterm atom. Under the hypothesis that the interaction between the collider and the atom can be described by a dipolar operator, we provide expressions that relate the collisional rates for interference between different $J$-levels to the usual collisional rates for $J$-level populations, for which experimental data or approximate theoretical expressions are generally available. We show that the rates for populations and interference within the same $J$-level reduce to those previously obtained for the multilevel model atom (where quantum interference is assumed to be present only between magnetic sublevels pertaining to any given $J$-level). Finally, we apply the general equations to the case of a two-term atom with unpolarized lower term, illustrating the impact of inelastic and superelastic collisions on the scattering line polarization through radiative transfer calculations in a slab of stellar atmospheric plasma anisotropically illuminated by the photospheric radiation field.
\end{abstract}

Key words. atomic processes - line: formation - polarization - radiative transfer - scattering - stars: atmospheres

\section{Introduction}

The intensity and polarization of the spectral line radiation emerging from an astrophysical plasma depends on the population and atomic polarization (i.e., population imbalances and quantum interference between different magnetic sublevels) of the lower and upper line levels at each spatial point along the line of sight (LOS). Determining the population and atomic polarization of such levels requires considering the interactions between the atoms and photons (radiative transitions) and between the atoms and other material particles, such as electrons, atoms, and ions (collisional transitions). This problem can be very complex, especially when it comes to modeling the spectral line polarization produced by the joint action of anisotropic radiation pumping and the Hanle and Zeeman effects in multilevel systems.

Within the framework of the density-matrix theory of spectral line polarization described in the monograph by Landi Degl'Innocenti \& Landolfi (2004; hereafter LL04), it is possible to develop a consistent set of equations for multilevel systems, either by neglecting (multilevel model atom) or considering (multiterm model atom) quantum interference between pairs of magnetic sublevels pertaining to different $J$-levels (with $J$ the level's total angular momentum value). The relevant equations are the radiative transfer equation for the Stokes parameters (where the coefficients of the emission vector and of the propagation matrix depend on the values of the atomic density matrix) and the master equation for the atomic density matrix (which includes both radiative and collisional rates).

While for the multilevel model atom LL04 derived the expressions for both radiative and collisional rates (assuming isotropic collisions), for the multiterm model atom they only provide the expressions for the radiative rates. The aim of this paper is to formally define the collisional rates for a multiterm atom, and to find their relevant properties, focusing our attention only on isotropic inelastic and superelastic collisions. The treatment of elastic collisions in a multiterm atom is actually more complicated, and will not be treated here. Such collisions (e.g., with neutral hydrogen atoms) tend to equalize the populations of the sublevels pertaining to any given $J$-level and to destroy any quantum interference between pairs of them. A similar depolarizing role may be caused by inelastic and superelastic collisions between the $J$-levels pertaining to any given term, especially when such $J$-levels are 
very close in energy (see Bommier 2009, for the hydrogen case). For the sake of simplicity, the latter type of collision will also be neglected, the investigation being limited to inelastic and superelastic collisions between different terms.

In the main body of this paper, we provide suitable expressions for the transfer and relaxation rates caused by isotropic inelastic and superelastic collisions, taking the possibility of atomic polarization in all the terms of the model atom into account. Particular attention is given to the collisional transfer and relaxation rates for interference between magnetic sublevels pertaining to different $J$-levels, the physical ingredient that cannot be accounted for with a multilevel model atom. Since there are basically no experimental data for such rates, we provide approximate expressions that relate such rates to the usual collisional rates that describe transitions between different $J$-levels (for which experimental data or theoretical expressions are generally available). As a consistency proof of our derivation, we show that the transfer and relaxation rates for populations and interference between pairs of magnetic sublevels pertaining to the same $J$-level reduce to those derived by LL04 for the multilevel atom case.

In the last section we present an illustrative application of the theoretical scheme developed here. We consider a two-term atom with unpolarized lower term, and we show the sensitivity to the collisional rates of the linear polarization of the radiation emerging from a slab of given optical depth, located at a given height above the "surface" of a solar-like star, and illuminated by its photospheric radiation field.

\section{Transfer rate due to inelastic collisions}

We consider a multiterm atom (see Sects. 7.5 and 7.6 of LL04) in the absence of magnetic fields, and we describe it by means of the density matrix elements $\rho_{\beta L S}\left(J M, J^{\prime} M^{\prime}\right)$, with $J$ the total angular momentum, $M$ its projection along the quantization axis, $L$ the orbital angular momentum, $S$ the spin, and $\beta$ the electronic configuration. This atomic model accounts for quantum interference (or coherence) between pairs of magnetic sublevels pertaining either to the same $J$-level or to different $J$-levels of the same term ( $J$-state interference). We also work using the multipole moments of the density matrix (or spherical statistical tensors), defined by the equation

$\beta L S \rho_{Q}^{K}\left(J, J^{\prime}\right)=\sum_{M M^{\prime}}(-1)^{J-M} \sqrt{2 K+1}\left(\begin{array}{ccc}J & J^{\prime} & K \\ M & -M^{\prime} & -Q\end{array}\right) \rho_{\beta L S}\left(J M, J^{\prime} M^{\prime}\right)$

Although collisional processes can be very efficient in coupling $J$-levels pertaining to the same term, in this investigation we only consider collisional processes coupling populations and coherence pertaining to different terms.

In a given, although arbitrary, reference system, transfer processes due to inelastic collisions contribute to the time evolution of a particular density matrix element according to the equation

$\frac{\mathrm{d}}{\mathrm{d} t} \rho_{\beta L S}\left(J M, J^{\prime} M^{\prime}\right)=\sum_{\beta_{\ell} L_{\ell} J_{\ell} M_{\ell} J_{\ell}^{\prime} M_{\ell}^{\prime}} C_{I}\left(\beta L S J M J^{\prime} M^{\prime}, \beta_{\ell} L_{\ell} S J_{\ell} M_{\ell} J_{\ell}^{\prime} M_{\ell}^{\prime}\right) \rho_{\beta_{\ell} L_{\ell} S}\left(J_{\ell} M_{\ell}, J_{\ell}^{\prime} M_{\ell}^{\prime}\right)$

where $C_{I}$ is the inelastic collision transfer rate and where the quantum numbers $\left(\beta_{\ell} L_{\ell} S\right)$ denote any term having energy lower than the term $(\beta L S)^{1}$. In a new reference system, obtained from the old one by the rotation $R$, recalling the transformation law (see Eq. (3.95) of LL04)

$\left[\rho_{\beta L S}\left(J M, J^{\prime} M^{\prime}\right)\right]_{\text {new }}=\sum_{N N^{\prime}} \mathcal{D}_{N M}^{J}(R)^{*} \mathcal{D}_{N^{\prime} M^{\prime}}^{J^{\prime}}(R)\left[\rho_{\beta L S}\left(J N, J^{\prime} N^{\prime}\right)\right]_{\mathrm{old}}$

with $\mathcal{D}_{M N}^{J}(R)$ the rotation matrices, and its inverse

$\left[\rho_{\beta L S}\left(J M, J^{\prime} M^{\prime}\right)\right]_{\mathrm{old}}=\sum_{N N^{\prime}} \mathcal{D}_{M N}^{J}(R) \mathcal{D}_{M^{\prime} N^{\prime}}^{J^{\prime}}(R)^{*}\left[\rho_{\beta L S}\left(J N, J^{\prime} N^{\prime}\right)\right]_{\mathrm{new}}$,

we have

$$
\begin{aligned}
\frac{\mathrm{d}}{\mathrm{d} t}\left[\rho_{\beta L S}\left(J M, J^{\prime} M^{\prime}\right)\right]_{\mathrm{new}}= & \sum_{\beta_{\ell} L_{\ell} J_{\ell} M_{\ell} J_{\ell}^{\prime} M_{\ell}^{\prime}} \\
& \left\{\sum_{N N^{\prime} N_{\ell} N_{\ell}^{\prime}} \mathcal{D}_{N M}^{J}(R)^{*} \mathcal{D}_{N^{\prime} M^{\prime}}^{J^{\prime}}(R) \mathcal{D}_{N_{\ell} M_{\ell}}^{J_{\ell}}(R) \mathcal{D}_{N_{\ell}^{\prime} M_{\ell}^{\prime}}^{J_{\ell}^{\prime}}(R)^{*} C_{I}\left(\beta L S J N J^{\prime} N^{\prime}, \beta_{\ell} L_{\ell} S J_{\ell} N_{\ell} J_{\ell}^{\prime} N_{\ell}^{\prime}\right)\right\} \\
\times & {\left[\rho_{\beta_{\ell} L_{\ell} S}\left(J_{\ell} M_{\ell}, J_{\ell}^{\prime} M_{\ell}^{\prime}\right)\right]_{\text {new }} }
\end{aligned}
$$

The assumption of isotropic collisions implies that all the quantization directions are equivalent, so that Eqs. (2) and (5) have to be identical. It follows that the collisional rates must satisfy the relation

$C_{I}\left(\beta L S J M J^{\prime} M^{\prime}, \beta_{\ell} L_{\ell} S J_{\ell} M_{\ell} J_{\ell}^{\prime} M_{\ell}^{\prime}\right)=\sum_{N N^{\prime} N_{\ell} N_{\ell}^{\prime}} \mathcal{D}_{N M}^{J}(R)^{*} \mathcal{D}_{N^{\prime} M^{\prime}}^{J^{\prime}}(R) \mathcal{D}_{N_{\ell} M_{\ell}}^{J_{\ell}}(R) \mathcal{D}_{N_{\ell}^{\prime} M_{\ell}^{\prime}}^{J_{\ell}^{\prime}}(R)^{*} C_{I}\left(\beta L S J N J^{\prime} N^{\prime}, \beta_{\ell} L_{\ell} S J_{\ell} N_{\ell} J_{\ell}^{\prime} N_{\ell}^{\prime}\right)$.

1 We assume that there is no overlapping in energy among the various terms of the model atom under consideration. 
After coupling through Eq. (A.10) the rotation matrices $\mathcal{D}_{N M}^{J}(R)^{*}$ and $\mathcal{D}_{N^{\prime} M^{\prime}}^{J^{\prime}}(R)$, as well as the rotation matrices $\mathcal{D}_{N_{\ell} M_{\ell}}^{J_{\ell}}(R)^{*}$ and $\mathcal{D}_{N_{\ell}^{\prime} M_{\ell}^{\prime}}^{J_{t}^{\prime}}(R)$, and using the complex conjugate of Eq. (A.11) on the ensuing expression, Eq. (6) takes the form

$$
\begin{aligned}
& C_{I}\left(\beta L S J M J^{\prime} M^{\prime}, \beta_{\ell} L_{\ell} S J_{\ell} M_{\ell} J_{\ell}^{\prime} M_{\ell}^{\prime}\right)=\sum_{N N^{\prime} N_{\ell} N_{\ell}^{\prime}} C_{I}\left(\beta L S J N J^{\prime} N^{\prime}, \beta_{\ell} L_{\ell} S J_{\ell} N_{\ell} J_{\ell}^{\prime} N_{\ell}^{\prime}\right) \\
& \times \sum_{K K^{\prime} K^{\prime \prime}}(-1)^{N-M+N_{\ell}^{\prime}-M_{\ell}^{\prime}}(2 K+1)\left(2 K^{\prime}+1\right)\left(2 K^{\prime \prime}+1\right) \\
& \times\left(\begin{array}{ccc}
J^{\prime} & J & K \\
N^{\prime} & -N & P
\end{array}\right)\left(\begin{array}{ccc}
J^{\prime} & J & K \\
M^{\prime} & -M & Q
\end{array}\right)\left(\begin{array}{ccc}
J_{\ell} & J_{\ell}^{\prime} & K^{\prime} \\
N_{\ell} & -N_{\ell}^{\prime} & P^{\prime}
\end{array}\right)\left(\begin{array}{ccc}
J_{\ell} & J_{\ell}^{\prime} & K^{\prime} \\
M_{\ell} & -M_{\ell}^{\prime} & Q^{\prime}
\end{array}\right) \\
& \times\left(\begin{array}{lll}
K & K^{\prime} & K^{\prime \prime} \\
P & P^{\prime} & P^{\prime \prime}
\end{array}\right)\left(\begin{array}{lll}
K & K^{\prime} & K^{\prime \prime} \\
Q & Q^{\prime} & Q^{\prime \prime}
\end{array}\right) \mathcal{D}_{P^{\prime \prime} Q^{\prime \prime}}^{K^{\prime \prime}}(R) .
\end{aligned}
$$

As the righthand side of Eq. (7) must be independent of the rotation $R$, the index $K^{\prime \prime}$ can only take the value $K^{\prime \prime}=0$. This implies $K=K^{\prime}, P=-P^{\prime}$, and $Q=-Q^{\prime}$. Using Eq. (A.4), we obtain

$$
\begin{aligned}
C_{I}\left(\beta L S J M J^{\prime} M^{\prime}, \beta_{\ell} L_{\ell} S J_{\ell} M_{\ell} J_{\ell}^{\prime} M_{\ell}^{\prime}\right)= & \sum_{N N^{\prime} N_{\ell} N_{\ell}^{\prime}} C_{I}\left(\beta L S J N J^{\prime} N^{\prime}, \beta_{\ell} L L_{\ell} S J_{\ell} N_{\ell} J_{\ell}^{\prime} N_{\ell}^{\prime}\right) \sum_{K}(2 K+1)(-1)^{N-M+N_{\ell}^{\prime}-M_{\ell}^{\prime}-P-Q} \\
& \times\left(\begin{array}{ccc}
J^{\prime} & J & K \\
N^{\prime} & -N & P
\end{array}\right)\left(\begin{array}{ccc}
J^{\prime} & J & K \\
M^{\prime} & -M & Q
\end{array}\right)\left(\begin{array}{ccc}
J_{\ell} & J_{\ell}^{\prime} & K \\
N_{\ell} & -N_{\ell}^{\prime} & -P
\end{array}\right)\left(\begin{array}{ccc}
J_{\ell} & J_{\ell}^{\prime} & K \\
M_{\ell} & -M_{\ell}^{\prime} & -Q
\end{array}\right) .
\end{aligned}
$$

\subsection{Multipole components of the inelastic collision transfer rate}

Introducing the multipole components of the inelastic collision transfer rate, defined by the equation ${ }^{2}$

$$
\begin{aligned}
C_{I}^{(K)}\left(\beta L S J J^{\prime}, \beta_{\ell} L_{\ell} S J_{\ell} J_{\ell}^{\prime}\right)= & \sqrt{\frac{J+J^{\prime}+1}{J_{\ell}+J_{\ell}^{\prime}+1}} \\
& \times \sum_{N N^{\prime} N_{\ell} N_{\ell}^{\prime}}(-1)^{J+J_{\ell}-N^{\prime}-N_{\ell}^{\prime}}\left(\begin{array}{ccc}
J^{\prime} & J & K \\
N^{\prime} & -N & P
\end{array}\right)\left(\begin{array}{ccc}
J_{\ell}^{\prime} & J_{\ell} & K \\
N_{\ell}^{\prime} & -N_{\ell} & P
\end{array}\right) C_{I}\left(\beta L S J N J^{\prime} N^{\prime}, \beta_{\ell} L_{\ell} S J_{\ell} N_{\ell} J_{\ell}^{\prime} N_{\ell}^{\prime}\right),
\end{aligned}
$$

and making use of Eqs. (A.1) and (A.2), Eq. (8) can be written in the form

$$
\begin{aligned}
C_{I}\left(\beta L S J M J^{\prime} M^{\prime}, \beta_{\ell} L_{\ell} S J_{\ell} M_{\ell} J_{\ell}^{\prime} M_{\ell}^{\prime}\right)= & \sqrt{\frac{J_{\ell}+J_{\ell}^{\prime}+1}{J+J^{\prime}+1}}(-1)^{J+J_{\ell}-M^{\prime}-M_{\ell}^{\prime}} \\
& \times \sum_{K}(2 K+1)\left(\begin{array}{ccc}
J^{\prime} & J & K \\
M^{\prime} & -M & Q
\end{array}\right)\left(\begin{array}{ccc}
J_{\ell}^{\prime} & J_{\ell} & K \\
M_{\ell}^{\prime} & -M_{\ell} & Q
\end{array}\right) C_{I}^{(K)}\left(\beta L S J J^{\prime}, \beta_{\ell} L_{\ell} S J_{\ell} J_{\ell}^{\prime}\right) .
\end{aligned}
$$

Substituting Eq. (10) into Eq. (2), and recalling the definition of the multipole moments of the density matrix (see Eq. (1)), with the help of Eq. (A.3), we find the following equation for the spherical statistical tensors

$\frac{\mathrm{d}}{\mathrm{d} t} \beta L S \rho_{Q}^{K}\left(J, J^{\prime}\right)=\sum_{\beta_{\ell} L_{\ell} J_{\ell} J_{\ell}^{\prime}} \sqrt{\frac{J_{\ell}+J_{\ell}^{\prime}+1}{J+J^{\prime}+1}} C_{I}^{(K)}\left(\beta L S J J^{\prime}, \beta_{\ell} L_{\ell} S J_{\ell} J_{\ell}^{\prime}\right) \beta_{\ell} L_{\ell} S \rho_{Q}^{K}\left(J_{\ell}, J_{\ell}^{\prime}\right)$.

Taking the complex conjugate of Eq. (2) and recalling that $\rho_{\beta L S}\left(J M, J^{\prime} M^{\prime}\right)^{*}=\rho_{\beta L S}\left(J^{\prime} M^{\prime}, J M\right)$, we have

$C_{I}\left(\beta L S J M J^{\prime} M^{\prime}, \beta_{\ell} L_{\ell} S J_{\ell} M_{\ell} J_{\ell}^{\prime} M_{\ell}^{\prime}\right)^{*}=C_{I}\left(\beta L S J^{\prime} M^{\prime} J M, \beta_{\ell} L_{\ell} S J_{\ell}^{\prime} M_{\ell}^{\prime} J_{\ell} M_{\ell}\right)$,

and therefore, using Eqs. (A.1) and (A.2),

$C_{I}^{(K)}\left(\beta L S J J^{\prime}, \beta_{\ell} L_{\ell} S J_{\ell} J_{\ell}^{\prime}\right)^{*}=(-1)^{J+J_{\ell}-J^{\prime}-J_{\ell}^{\prime}} C_{I}^{(K)}\left(\beta L S J^{\prime} J, \beta_{\ell} L_{\ell} S J_{\ell}^{\prime} J_{\ell}\right)$.

Setting $K=0$ in Eq. (9), and using Eq. (A.4), we obtain

$C_{I}^{(0)}\left(\beta L S J J^{\prime}, \beta_{\ell} L_{\ell} S J_{\ell} J_{\ell}^{\prime}\right)=\delta_{J J^{\prime}} \delta_{J_{\ell} J_{\ell}} \frac{1}{2 J_{\ell}+1} \sum_{N N_{\ell}} C_{I}\left(\beta L S J N J N, \beta_{\ell} L_{\ell} S J_{\ell} N_{\ell} J_{\ell} N_{\ell}\right)$,

where the transfer rate $C_{I}\left(\beta L S J N J N, \beta_{\ell} L_{\ell} S J_{\ell} N_{\ell} J_{\ell} N_{\ell}\right)$ is the usual (inelastic) collisional rate for the transition from the lower magnetic sublevel $\left|\beta_{\ell} L_{\ell} S J_{\ell} N_{\ell}\right\rangle$ to the upper magnetic sublevel $|\beta L S J N\rangle$, generally indicated in the literature with the notation $C_{I}\left(\beta_{\ell} L_{\ell} S J_{\ell} N_{\ell} \rightarrow \beta L S J N\right)$. Since this rate is non-negative, the 0-rank multipole component is also non-negative.

2 The factor $\sqrt{J+J^{\prime}+1} / \sqrt{J_{\ell}+J_{\ell}^{\prime}+1}$ is introduced in order to get simpler relations between these rates and the usual collisional rates connecting atomic populations. This factor reduces to the one introduced in the multilevel atom case (see Eq. (7.87) of LL04) when interference between different $J$-levels is neglected $\left(J=J^{\prime}\right.$ and $\left.J_{\ell}=J_{\ell}^{\prime}\right)$. 


\subsection{Relations with the collisional rates for J-level populations}

In most cases, the only collisional rates for which experimental data, or approximate analytical expressions, are available are the collisional rates connecting the populations of different $J$-levels (following the notation generally used in the literature, these rates will be indicated through the symbols $C_{I}\left(\beta_{\ell} L_{\ell} S J_{\ell} \rightarrow \beta_{u} L_{u} S J_{u}\right)$ and $C_{S}\left(\beta_{u} L_{u} S J_{u} \rightarrow \beta_{\ell} L_{\ell} S J_{\ell}\right)$, the indices $I$ and $S$ standing for "inelastic" and "superelastic", respectively). It is important therefore to find suitable relations between such rates and the collisional rates introduced in this paper for a multiterm atom.

Observing that

$C_{I}\left(\beta_{\ell} L_{\ell} S J_{\ell} \rightarrow \beta_{u} L_{u} S J_{u}\right)=\frac{1}{2 J_{\ell}+1} \sum_{N_{u} N_{\ell}} C_{I}\left(\beta_{\ell} L_{\ell} S J_{\ell} N_{\ell} \rightarrow \beta_{u} L_{u} S J_{u} N_{u}\right)$,

from Eq. (14) we immediately have

$C_{I}^{(0)}\left(\beta L S J J, \beta_{\ell} L_{\ell} S J_{\ell} J_{\ell}\right)=C_{I}\left(\beta_{\ell} L_{\ell} S J_{\ell} \rightarrow \beta L S J\right)$

In Eq. (6), if we couple through Eq. (A.10) the rotation matrices $\mathcal{D}_{N M}^{J}(R)^{*}$ and $\mathcal{D}_{N_{\ell} M_{\ell}}^{J_{\ell}}(R)$, as well as the rotation matrices $\mathcal{D}_{N^{\prime} M^{\prime}}^{J^{\prime}}(R)$ and $\mathcal{D}_{N_{\ell}^{\prime} M_{\ell}^{\prime}}^{J^{\prime}}(R)^{*}$, by requiring that the ensuing expression is independent of the rotation $R$, we find the relation

$$
\begin{aligned}
C_{I}\left(\beta L S J M J^{\prime} M^{\prime}, \beta_{\ell} L_{\ell} S J_{\ell} M_{\ell} J_{\ell}^{\prime} M_{\ell}^{\prime}\right)= & \sum_{N N^{\prime} N_{\ell} N_{\ell}^{\prime}} C_{I}\left(\beta L S J N J^{\prime} N^{\prime}, \beta_{\ell} L_{\ell} S J_{\ell} N_{\ell} J_{\ell}^{\prime} N_{\ell}^{\prime}\right) \sum_{K}(2 K+1)(-1)^{N-M+N_{\ell}^{\prime}-M_{\ell}^{\prime}-P-Q} \\
& \times\left(\begin{array}{ccc}
J & J_{\ell} & K \\
-M & M_{\ell} & Q
\end{array}\right)\left(\begin{array}{ccc}
J^{\prime} & J_{\ell}^{\prime} & K \\
-M^{\prime} & M_{\ell}^{\prime} & Q
\end{array}\right)\left(\begin{array}{ccc}
J & J_{\ell} & K \\
-N & N_{\ell} & P
\end{array}\right)\left(\begin{array}{ccc}
J^{\prime} & J_{\ell}^{\prime} & K \\
-N^{\prime} & N_{\ell}^{\prime} & P
\end{array}\right) .
\end{aligned}
$$

Defining a different set of multipole components of the inelastic collision transfer rate through the equation

$$
\Gamma_{I}^{(K)}\left(\beta L S J J^{\prime}, \beta_{\ell} L_{\ell} S J_{\ell} J_{\ell}^{\prime}\right)=\frac{(2 K+1)}{J_{\ell}+J_{\ell}^{\prime}+1} \sum_{N N^{\prime} N_{\ell} N_{\ell}^{\prime}}(-1)^{N_{\ell}^{\prime}-N_{\ell}}\left(\begin{array}{ccc}
J & J_{\ell} & K \\
-N & N_{\ell} & P
\end{array}\right)\left(\begin{array}{ccc}
J^{\prime} & J_{\ell}^{\prime} & K \\
-N^{\prime} & N_{\ell}^{\prime} & P
\end{array}\right) C_{I}\left(\beta L S J N J^{\prime} N^{\prime}, \beta_{\ell} L_{\ell} S J_{\ell} N_{\ell} J_{\ell}^{\prime} N_{\ell}^{\prime}\right)
$$

Equation (17) can be written in the form

$$
C_{I}\left(\beta L S J M J^{\prime} M^{\prime}, \beta_{\ell} L_{\ell} S J_{\ell} M_{\ell} J_{\ell}^{\prime} M_{\ell}^{\prime}\right)=(-1)^{M_{\ell}^{\prime}-M_{\ell}}\left(J_{\ell}+J_{\ell}^{\prime}+1\right) \sum_{K}\left(\begin{array}{ccc}
J & J_{\ell} & K \\
-M & M_{\ell} & Q
\end{array}\right)\left(\begin{array}{ccc}
J^{\prime} & J_{\ell}^{\prime} & K \\
-M^{\prime} & M_{\ell}^{\prime} & Q
\end{array}\right) \Gamma_{I}^{(K)}\left(\beta L S J J^{\prime}, \beta_{\ell} L_{\ell} S J_{\ell} J_{\ell}^{\prime}\right)
$$

As pointed out in LL04 for the multilevel atom case, this decomposition of the collisional rate has an interesting physical interpretation, because it shows that the interaction between the atomic system and the collider can be described by a sum of tensor operators of rank $K$ acting on the state vectors of the atom. Starting from Eq. (9) and using Eq. (A.8), after some algebra the following relation between the multipole components $C_{I}^{(K)}$ and $\Gamma_{I}^{(K)}$ can be found:

$C_{I}^{(K)}\left(\beta L S J J^{\prime}, \beta_{\ell} L_{\ell} S J_{\ell} J_{\ell}^{\prime}\right)=\sqrt{\left(J+J^{\prime}+1\right)\left(J_{\ell}+J_{\ell}^{\prime}+1\right)} \sum_{K^{\prime}}(-1)^{J^{\prime}+J_{\ell}^{\prime}-K^{\prime}+K}\left\{\begin{array}{lll}J^{\prime} & J & K \\ J_{\ell} & J_{\ell}^{\prime} & K^{\prime}\end{array}\right\} \Gamma_{I}^{\left(K^{\prime}\right)}\left(\beta L S J J^{\prime}, \beta_{\ell} L_{\ell} S J_{\ell} J_{\ell}^{\prime}\right)$

For the $K=0$ multipole component, using Eq. (A.7), we have (cf. Appendix A4 of LL04)

$C_{I}^{(0)}\left(\beta L S J J, \beta_{\ell} L_{\ell} S J_{\ell} J_{\ell}\right)=\sum_{K} \Gamma_{I}^{(K)}\left(\beta L S J J, \beta_{\ell} L_{\ell} S J_{\ell} J_{\ell}\right)$

When the interaction can be described through just one operator of $\operatorname{rank} \tilde{K}$, then

$C_{I}^{(K)}\left(\beta L S J J^{\prime}, \beta_{\ell} L_{\ell} S J_{\ell} J_{\ell}^{\prime}\right)=\sqrt{\left(J+J^{\prime}+1\right)\left(J_{\ell}+J_{\ell}^{\prime}+1\right)}(-1)^{J^{\prime}+J_{\ell}^{\prime}-\tilde{K}+K}\left\{\begin{array}{lll}J^{\prime} & J & K \\ J_{\ell} & J_{\ell}^{\prime} & \tilde{K}\end{array}\right\} \Gamma_{I}^{(\tilde{K})}\left(\beta L S J J^{\prime}, \beta_{\ell} L_{\ell} S J_{\ell} J_{\ell}^{\prime}\right)$

The multipole component of rank $K$ of the diagonal rates $\left(J=J^{\prime}\right.$ and $\left.J_{\ell}=J_{\ell}^{\prime}\right)$ is thus related to the multipole component of rank 0 by the equation (cf. Appendix A4 of LL04)

$C_{I}^{(K)}\left(\beta L S J J, \beta_{\ell} L_{\ell} S J_{\ell} J_{\ell}\right)=(-1)^{K} \frac{\left\{\begin{array}{ccc}J & J & K \\ J_{\ell} & J_{\ell} & \tilde{K}\end{array}\right\}}{\left\{\begin{array}{lll}J & J & 0 \\ J_{\ell} & J_{\ell} & \tilde{K}\end{array}\right\}} C_{I}^{(0)}\left(\beta L S J J, \beta_{\ell} L_{\ell} S J_{\ell} J_{\ell}\right)=(-1)^{K} \frac{\left\{\begin{array}{ccc}J & J & K \\ J_{\ell} & J_{\ell} & \tilde{K}\end{array}\right\}}{\left\{\begin{array}{ccc}J & J & 0 \\ J_{\ell} & J_{\ell} & \tilde{K}\end{array}\right\}} C_{I}\left(\beta_{\ell} L_{\ell} S J_{\ell} \rightarrow \beta L S J\right)$

A similar relation for the nondiagonal rates (describing the transfer of $J$-state interference due to inelastic collisions) cannot be obtained through symmetry arguments alone. Such a relation can, however, be derived if some simplifying hypotheses on the interaction between the atoms and perturbers are introduced. It is well known that in the case of electrons with much higher energy than the threshold energy (i.e. under the so-called Born approximation), the Hamiltonian describing the electron-atom interaction 
depends on the dynamical variables of the atom only through the dipole operator (a tensor of $\operatorname{rank} \tilde{K}=1$ ). A collisional process in an optically allowed transition can thus be treated, in a first approximation, as a radiative transition, and the collisional rate can be expressed through the oscillator strength of the same transition (e.g. Seaton 1962; Van Regemorter 1962).

For more insight on the nondiagonal rates $C_{I}^{(K)}\left(\beta L S J J^{\prime}, \beta_{\ell} L_{\ell} S J_{\ell} J_{\ell}^{\prime}\right)$, we assume that the electron-atom interaction is described by a dipolar operator, and we proceed by analogy with the multiterm atom radiative transfer rate due to absorption processes $\left(\mathbb{T}_{A}\right)$. Setting $K_{r}=0$ (i.e. assuming an isotropic radiation field) in Eq. (7.45a) of LL04, we have

$$
\begin{aligned}
\mathbb{T}_{A}\left(\beta L S K Q J J^{\prime}, \beta_{\ell} L_{\ell} S K Q J_{\ell} J_{\ell}^{\prime}\right)= & \left(2 L_{\ell}+1\right)(-1)^{1+J^{\prime}+J_{\ell}^{\prime}+K} \sqrt{(2 J+1)\left(2 J^{\prime}+1\right)\left(2 J_{\ell}+1\right)\left(2 J_{\ell}^{\prime}+1\right)} \\
& \times\left\{\begin{array}{ccc}
J & J_{\ell} & 1 \\
J_{\ell}^{\prime} & J^{\prime} & K
\end{array}\right\}\left\{\begin{array}{ccc}
L & L_{\ell} & 1 \\
J_{\ell} & J & S
\end{array}\right\}\left\{\begin{array}{ccc}
L & L_{\ell} & 1 \\
J_{\ell}^{\prime} & J^{\prime} & S
\end{array}\right\} B\left(\beta_{\ell} L_{\ell} S \rightarrow \beta L S\right) J_{0}^{0},
\end{aligned}
$$

where $J_{0}^{0}$ is the angle-averaged incident radiation field, and $B\left(\beta_{\ell} L_{\ell} S \rightarrow \beta L S\right)$ is the Einstein coefficient for absorption from the lower term $\left(\beta_{\ell} L_{\ell} S\right)$ to the upper term $(\beta L S)$. We recall that this quantity is connected to the Einstein coefficients for the individual transitions between fine structure $J$-levels of the multiplet by the relation (see Eq. (7.57a) of LL04)

$B\left(\beta_{\ell} L_{\ell} S J_{\ell} \rightarrow \beta L S J\right)=\left(2 L_{\ell}+1\right)(2 J+1)\left\{\begin{array}{ccc}L & L_{\ell} & 1 \\ J_{\ell} & J & S\end{array}\right\}^{2} B\left(\beta_{\ell} L_{\ell} S \rightarrow \beta L S\right)$

which implies (using Eq. (A.5)) $)^{3}$

$B\left(\beta_{\ell} L_{\ell} S \rightarrow \beta L S\right)=\sum_{J} B\left(\beta_{\ell} L_{\ell} S J_{\ell} \rightarrow \beta L S J\right)$

By analogy with Eq. (26), we define an inelastic collisional rate for the transition from the lower to the upper term $C_{I}\left(\beta_{\ell} L_{\ell} S \rightarrow \beta L S\right)$ through the equation

$C_{I}\left(\beta_{\ell} L_{\ell} S \rightarrow \beta L S\right)=\sum_{J} C_{I}\left(\beta_{\ell} L_{\ell} S J_{\ell} \rightarrow \beta L S J\right)$

where the sum is extended to all the $J$-levels of the upper term to which a given $J$-level of the lower term can be connected through an electric dipole transition. By analogy with Eq. (24), and taking the multiplying factor introduced in Eq. (9) into account (see footnote 2), we can write

$$
\begin{array}{r}
C_{I}^{(K)}\left(\beta L S J J^{\prime}, \beta_{\ell} L_{\ell} S J_{\ell} J_{\ell}^{\prime}\right)=\left(2 L_{\ell}+1\right)(-1)^{1+J^{\prime}+J_{\ell}^{\prime}+K} \sqrt{\frac{\left(J+J^{\prime}+1\right)(2 J+1)\left(2 J^{\prime}+1\right)\left(2 J_{\ell}+1\right)\left(2 J_{\ell}^{\prime}+1\right)}{J_{\ell}+J_{\ell}^{\prime}+1}} \\
\times\left\{\begin{array}{ccc}
J & J_{\ell} & 1 \\
J_{\ell}^{\prime} & J^{\prime} & K
\end{array}\right\}\left\{\begin{array}{ccc}
L & L_{\ell} & 1 \\
J_{\ell} & J & S
\end{array}\right\}\left\{\begin{array}{ccc}
L & L_{\ell} & 1 \\
J_{\ell}^{\prime} & J^{\prime} & S
\end{array}\right\} C_{I}\left(\beta_{\ell} L_{\ell} S \rightarrow \beta L S\right) .
\end{array}
$$

This equation can be used to calculate the multipole components of the inelastic collision transfer rates for $J$-state interference from the values of the usual inelastic collisional rates for $J$-level populations. As a proof of the consistency of Eq. (28), we observe that the 0-rank multipole component is given by

$C_{I}^{(0)}\left(\beta L S J J, \beta_{\ell} L_{\ell} S J_{\ell} J_{\ell}\right)=C_{I}\left(\beta_{\ell} L_{\ell} S J_{\ell} \rightarrow \beta L S J\right)=\left(2 L_{\ell}+1\right)(2 J+1)\left\{\begin{array}{ccc}L & L_{\ell} & 1 \\ J_{\ell} & J & S\end{array}\right\}^{2} C_{I}\left(\beta_{\ell} L_{\ell} S \rightarrow \beta L S\right)$,

which is the analogous to Eq. (25), while using Eqs. (A.7) and (29), the diagonal terms are given by

$C_{I}^{(K)}\left(\beta L S J J, \beta_{\ell} L_{\ell} S J_{\ell} J_{\ell}\right)=(-1)^{K} \frac{\left\{\begin{array}{ccc}J & J & K \\ J_{\ell} & J_{\ell} & 1\end{array}\right\}}{\left\{\begin{array}{ccc}J & J & 0 \\ J_{\ell} & J_{\ell} & 1\end{array}\right\}} C_{I}\left(\beta_{\ell} L_{\ell} S J_{\ell} \rightarrow \beta L S J\right)$,

which corresponds to Eq. (23) for $\tilde{K}=1$.

3 The sum appearing on the righthand side of Eq. (26) does not depend on the particular $J$-level of the lower term that is considered. 


\section{Transfer rate due to superelastic collisions}

A similar reasoning can be followed for the transfer rates due to superelastic collisions. These transfer processes contribute to the time evolution of a particular density matrix element according to the equation

$$
\frac{\mathrm{d}}{\mathrm{d} t} \rho_{\beta L S}\left(J M, J^{\prime} M^{\prime}\right)=\sum_{\beta_{u} L_{u} J_{u} M_{u} J_{u}^{\prime} M_{u}^{\prime}} C_{S}\left(\beta L S J M J^{\prime} M^{\prime}, \beta_{u} L_{u} S J_{u} M_{u} J_{u}^{\prime} M_{u}^{\prime}\right) \rho_{\beta_{u} L_{u} S}\left(J_{u} M_{u}, J_{u}^{\prime} M_{u}^{\prime}\right)
$$

where $C_{S}$ is the superelastic collision transfer rate and where the quantum numbers $\left(\beta_{u} L_{u} S\right)$ denote any term having energy higher than the term $(\beta L S)$. Following the same steps as in Sect. 2, it can be shown that under the assumption of isotropic collisions, the transfer rate $C_{S}$ can be written in the form

$$
\begin{aligned}
C_{S}\left(\beta L S J M J^{\prime} M^{\prime}, \beta_{u} L_{u} S J_{u} M_{u} J_{u}^{\prime} M_{u}^{\prime}\right)= & \sqrt{\frac{J_{u}+J_{u}^{\prime}+1}{J+J^{\prime}+1}}(-1)^{J+J_{u}-M^{\prime}-M_{u}^{\prime}} \\
& \times \sum_{K}(2 K+1)\left(\begin{array}{ccc}
J^{\prime} & J & K \\
M^{\prime} & -M & Q
\end{array}\right)\left(\begin{array}{ccc}
J_{u}^{\prime} & J_{u} & K \\
M_{u}^{\prime} & -M_{u} & Q
\end{array}\right) C_{S}^{(K)}\left(\beta L S J J^{\prime}, \beta_{u} L_{u} S J_{u} J_{u}^{\prime}\right),
\end{aligned}
$$

where the multipole components of the superelastic collision transfer rate, $C_{S}^{(K)}$, are defined by the equation

$$
\begin{aligned}
C_{S}^{(K)}\left(\beta L S J J^{\prime}, \beta_{u} L_{u} S J_{u} J_{u}^{\prime}\right)= & \sqrt{\frac{J+J^{\prime}+1}{J_{u}+J_{u}^{\prime}+1}} \\
& \times \sum_{N N^{\prime} N_{u} N_{u}^{\prime}}(-1)^{J+J_{u}-N^{\prime}-N_{u}^{\prime}}\left(\begin{array}{ccc}
J^{\prime} & J & K \\
N^{\prime} & -N & P
\end{array}\right)\left(\begin{array}{ccc}
J_{u}^{\prime} & J_{u} & K \\
N_{u}^{\prime} & -N_{u} & P
\end{array}\right) C_{S}\left(\beta L S J N J^{\prime} N^{\prime}, \beta_{u} L_{u} S J_{u} N_{u} J_{u}^{\prime} N_{u}^{\prime}\right)
\end{aligned}
$$

By substituting Eq. (32) into Eq. (31), and recalling Eq. (1), we find the following equation for the spherical statistical tensors

$$
\frac{\mathrm{d}}{\mathrm{d} t} \beta L S \rho_{Q}^{K}\left(J, J^{\prime}\right)=\sum_{\beta_{u} L_{u} J_{u} J_{u}^{\prime}} \sqrt{\frac{J_{u}+J_{u}^{\prime}+1}{J+J^{\prime}+1}} C_{S}^{(K)}\left(\beta L S J J^{\prime}, \beta_{u} L_{u} S J_{u} J_{u}^{\prime}\right){ }^{\beta_{u} L_{u} S} \rho_{Q}^{K}\left(J_{u}, J_{u}^{\prime}\right)
$$

The 0-rank multipole component is given by

$C_{S}^{(0)}\left(\beta L S J J^{\prime}, \beta_{u} L_{u} S J_{u} J_{u}^{\prime}\right)=\delta_{J J^{\prime}} \delta_{J_{u} J_{u}^{\prime}} \frac{1}{2 J_{u}+1} \sum_{N N_{u}} C_{S}\left(\beta L S J N J N, \beta_{u} L_{u} S J_{u} N_{u} J_{u} N_{u}\right)=\delta_{J J^{\prime}} \delta_{J_{u} J_{u}^{\prime}} C_{S}\left(\beta_{u} L_{u} S J_{u} \rightarrow \beta L S J\right)$,

where $C_{S}\left(\beta_{u} L_{u} S J_{u} \rightarrow \beta L S J\right)$ is the usual superelastic collisional rate for the transition from the upper level $\left|\beta_{u} L_{u} S J_{u}\right\rangle$ to the lower level $|\beta L S J\rangle$. When the interaction between the atomic system and the colliders can be described by means of a single operator of rank $\tilde{K}$, it can be shown that the multipole components of rank $K$ of the diagonal rates $\left(J=J^{\prime}\right.$ and $\left.J_{u}=J_{u}^{\prime}\right)$ are related to the multipole components of rank 0 by the equation

$$
C_{S}^{(K)}\left(\beta L S J J, \beta_{u} L_{u} S J_{u} J_{u}\right)=(-1)^{K} \frac{\left\{\begin{array}{ccc}
J & J & K \\
J_{u} & J_{u} & \tilde{K}
\end{array}\right\}}{\left\{\begin{array}{ccc}
J & J & 0 \\
J_{u} & J_{u} & \tilde{K}
\end{array}\right\}} C_{S}^{(0)}\left(\beta L S J J, \beta_{u} L_{u} S J_{u} J_{u}\right)=(-1)^{K} \frac{\left\{\begin{array}{ccc}
J & J & K \\
J_{u} & J_{u} & \tilde{K}
\end{array}\right\}}{\left\{\begin{array}{ccc}
J & J & 0 \\
J_{u} & J_{u} & \tilde{K}
\end{array}\right\}} C_{S}\left(\beta_{u} L_{u} S J_{u} \rightarrow \beta L S J\right) .
$$

As discussed in the previous section for the case of inelastic collisions, a similar relation for the nondiagonal rates (describing the transfer of $J$-state interference due to superelastic collisions) can be derived under the assumption that the electron-atom interaction is described by a dipolar operator. By analogy with the expression of the multiterm atom radiative transfer rate due to stimulated emission processes $\left(\mathbb{T}_{S}\right.$, see Eq. (7.45c) of LL04) in the presence of an isotropic incident field, we find the following relation

$$
\begin{aligned}
C_{S}^{(K)}\left(\beta L S J J^{\prime}, \beta_{u} L_{u} S J_{u} J_{u}^{\prime}\right)= & \left(2 L_{u}+1\right)(-1)^{1+J^{\prime}+J_{u}^{\prime}+K} \sqrt{\frac{\left(J+J^{\prime}+1\right)(2 J+1)\left(2 J^{\prime}+1\right)\left(2 J_{u}+1\right)\left(2 J_{u}^{\prime}+1\right)}{J_{u}+J_{u}^{\prime}+1}} \\
& \times\left\{\begin{array}{ccc}
J & J_{u} & 1 \\
J_{u}^{\prime} & J^{\prime} & K
\end{array}\right\}\left\{\begin{array}{ccc}
L & L_{u} & 1 \\
J_{u} & J & S
\end{array}\right\}\left\{\begin{array}{ccc}
L & L_{u} & 1 \\
J_{u}^{\prime} & J^{\prime} & S
\end{array}\right\} C_{S}\left(\beta_{u} L_{u} S \rightarrow \beta L S\right),
\end{aligned}
$$

where we have introduced the superelastic collisional rate for the transition from the upper to the lower term $C_{S}\left(\beta_{u} L_{u} S \rightarrow \beta L S\right)$, defined by

$C_{S}\left(\beta_{u} L_{u} S \rightarrow \beta L S\right)=\sum_{J} C_{S}\left(\beta_{u} L_{u} S J_{u} \rightarrow \beta L S J\right)$

the sum being extended to all the $J$-levels of the lower term to which a given $J$-level of the upper term can be connected through an electric dipole transition ${ }^{4}$.

4 The sum appearing on the righthand side of Eq. (38) does not depend on the particular $J$-level of the upper term that is considered. 


\section{Relaxation rates due to inelastic and superelastic collisions}

In a given reference system, relaxation processes due to inelastic and superelastic collisions contribute to the time evolution of a particular density-matrix element via an equation of the form

$\frac{\mathrm{d}}{\mathrm{d} t} \rho_{\beta L S}\left(J M, J^{\prime} M^{\prime}\right)=-\sum_{J^{\prime \prime} M^{\prime \prime}}\left[f\left(\beta L S J M J^{\prime} M^{\prime} J^{\prime \prime} M^{\prime \prime}\right) \rho_{\beta L S}\left(J M, J^{\prime \prime} M^{\prime \prime}\right)+g\left(\beta L S J M J^{\prime} M^{\prime} J^{\prime \prime} M^{\prime \prime}\right) \rho_{\beta L S}\left(J^{\prime \prime} M^{\prime \prime}, J^{\prime} M^{\prime}\right)\right]$.

The conjugation property of the density-matrix elements $\left(\rho_{\beta L S}\left(J M, J^{\prime} M^{\prime}\right)^{*}=\rho_{\beta L S}\left(J^{\prime} M^{\prime}, J M\right)\right)$ requires that

$g\left(\beta L S J M J^{\prime} M^{\prime} J^{\prime \prime} M^{\prime \prime}\right)=f\left(\beta L S J^{\prime} M^{\prime} J M J^{\prime \prime} M^{\prime \prime}\right)^{*}$,

so that Eq. (39) can be written in the form

$\frac{\mathrm{d}}{\mathrm{d} t} \rho_{\beta L S}\left(J M, J^{\prime} M^{\prime}\right)=-\sum_{J^{\prime \prime} M^{\prime \prime}}\left[\frac{1}{2} S\left(\beta L S J M J^{\prime} M^{\prime} J^{\prime \prime} M^{\prime \prime}\right) \rho_{\beta L S}\left(J M, J^{\prime \prime} M^{\prime \prime}\right)+\frac{1}{2} S\left(\beta L S J^{\prime} M^{\prime} J M J^{\prime \prime} M^{\prime \prime}\right)^{*} \rho_{\beta L S}\left(J^{\prime \prime} M^{\prime \prime}, J^{\prime} M^{\prime}\right)\right]$.

In a new reference system, obtained from the old one by the rotation $R$, recalling Eqs. (3) and (4), we have

$$
\begin{aligned}
\frac{\mathrm{d}}{\mathrm{d} t}\left[\rho_{\beta L S}\left(J M, J^{\prime} M^{\prime}\right)\right]_{\text {new }}=-\sum_{J^{\prime \prime} M^{\prime \prime} M^{\prime \prime \prime}}\left\{\frac{1}{2} \sum_{N N^{\prime} N^{\prime \prime}}\right. & \mathcal{D}_{N M}^{J}(R)^{*} \mathcal{D}_{N^{\prime} M^{\prime}}^{J^{\prime}}(R) S\left(\beta L S J N J^{\prime} N^{\prime} J^{\prime \prime} N^{\prime \prime}\right) \\
& \times \mathcal{D}_{N M^{\prime \prime \prime}}^{J}(R) \mathcal{D}_{N^{\prime \prime} M^{\prime \prime}}^{J^{\prime \prime}}(R)^{*}\left[\rho_{\beta L S}\left(J M^{\prime \prime \prime}, J^{\prime \prime} M^{\prime \prime}\right)\right]_{\text {new }} \\
+\frac{1}{2} \sum_{N N^{\prime} N^{\prime \prime}} & \mathcal{D}_{N M^{\prime}(R)^{*}}^{J} \mathcal{D}_{N^{\prime} M^{\prime}}^{J^{\prime}}(R) S\left(\beta L S J^{\prime} N^{\prime} J N J^{\prime \prime} N^{\prime \prime}\right)^{*} \\
& \left.\times \mathcal{D}_{N^{\prime \prime} M^{\prime \prime}}^{J^{\prime \prime}}(R) \mathcal{D}_{N^{\prime} M^{\prime \prime \prime}}^{J^{\prime \prime}}(R)^{*}\left[\rho_{\beta L S}\left(J^{\prime \prime} M^{\prime \prime}, J^{\prime} M^{\prime \prime \prime}\right)\right]_{\text {new }}\right\} .
\end{aligned}
$$

Due to the isotropy of collisions, Eqs. (41) and (42) must be identical, which implies

$S\left(\beta L S J M J^{\prime} M^{\prime} J^{\prime \prime} M^{\prime \prime}\right) \delta_{M M^{\prime \prime \prime}}=\sum_{N N^{\prime} N^{\prime \prime}} \mathcal{D}_{N M}^{J}(R)^{*} \mathcal{D}_{N^{\prime} M^{\prime}}^{J^{\prime}}(R) S\left(\beta L S J N J^{\prime} N^{\prime} J^{\prime \prime} N^{\prime \prime}\right) \mathcal{D}_{N M^{\prime \prime \prime}}^{J}(R) \mathcal{D}_{N^{\prime \prime} M^{\prime \prime}}^{J^{\prime \prime}}(R)^{*}$,

regardless of the rotation $R$. This requires the rate $S\left(\beta L S J N J^{\prime} N^{\prime} J^{\prime \prime} N^{\prime \prime}\right)$ to be independent of the quantum number $N$ (if not, the righthand side of Eq. (43) would not be zero for $M \neq M^{\prime \prime \prime}$, no matter the rotation $R$ ). We can thus carry out the summation over $N$ via Eq. (A.9) to get (with the help of Eq. (A.10))

$S\left(\beta L S J J^{\prime} M^{\prime} J^{\prime \prime} M^{\prime \prime}\right)=\sum_{N^{\prime} N^{\prime \prime}} S\left(\beta L S J J^{\prime} N^{\prime} J^{\prime \prime} N^{\prime \prime}\right)(-1)^{N^{\prime \prime}-M^{\prime \prime}} \sum_{K}(2 K+1)\left(\begin{array}{ccc}J^{\prime} & J^{\prime \prime} & K \\ N^{\prime} & -N^{\prime \prime} & P\end{array}\right)\left(\begin{array}{ccc}J^{\prime} & J^{\prime \prime} & K \\ M^{\prime} & -M^{\prime \prime} & Q\end{array}\right) \mathcal{D}_{P Q}^{K}(R)^{*}$.

Since the righthand side of Eq. (44) must be independent of the rotation $R$, index $K$ can only take the value $K=0$, which implies $Q=P=0, N^{\prime}=N^{\prime \prime}, M^{\prime}=M^{\prime \prime}$, and $J^{\prime}=J^{\prime \prime}$ from Eq. (A.4). We thus obtain

$S\left(\beta L S J J^{\prime} M^{\prime} J^{\prime \prime} M^{\prime \prime}\right)=\delta_{M^{\prime} M^{\prime \prime}} \delta_{J^{\prime} J^{\prime \prime}} \frac{1}{2 J^{\prime}+1} \sum_{N^{\prime}} S\left(\beta L S J J^{\prime} N^{\prime} J^{\prime} N^{\prime}\right)$.

Substitution into Eq. (41) gives

$\frac{\mathrm{d}}{\mathrm{d} t} \rho_{\beta L S}\left(J M, J^{\prime} M^{\prime}\right)=-S_{0}\left(\beta L S J J^{\prime}\right) \rho_{\beta L S}\left(J M, J^{\prime} M^{\prime}\right)$,

or, in the spherical statistical tensor representation,

$\frac{\mathrm{d}}{\mathrm{d} t} \beta L S \rho_{Q}^{K}\left(J, J^{\prime}\right)=-S_{0}\left(\beta L S J J^{\prime}\right)^{\beta L S} \rho_{Q}^{K}\left(J, J^{\prime}\right)$,

where we have introduced the collisional relaxation rate

$S_{0}\left(\beta L S J J^{\prime}\right)=\frac{1}{2}\left[\frac{1}{2 J^{\prime}+1} \sum_{M^{\prime}} S\left(\beta L S J J^{\prime} M^{\prime} J^{\prime} M^{\prime}\right)+\frac{1}{2 J+1} \sum_{M} S\left(\beta L S J^{\prime} J M J M\right)^{*}\right]$.

The diagonal element

$\left.S_{0}(\beta L S J J)=\frac{1}{2 J+1} \frac{1}{2} \sum_{M}\left[S(\beta L S J J M J M)+S(\beta L S J J M J M)^{*}\right]=\frac{1}{2 J+1} \operatorname{Re}\left[\sum_{M} S(\beta L S J J M J M)\right)\right]$

coincides with the one defined in LLO4 for the case of a multilevel atom. 
As shown in LL04, the diagonal element $S_{0}(\beta L S J J)$, which represents the relaxation rate of populations and interference between magnetic sublevels pertaining to the same $J$-level (see Eqs. (46) and (47)), is connected to the 0-rank multipole components of the inelastic and superelastic collision transfer rates by the equation

$S_{0}(\beta L S J J)=\sum_{\beta_{u} L_{u} J_{u}} C_{I}^{(0)}\left(\beta_{u} L_{u} S J_{u} J_{u}, \beta L S J J\right)+\sum_{\beta_{\ell} L_{\ell} J_{\ell}} C_{S}^{(0)}\left(\beta_{\ell} L_{\ell} S J_{\ell} J_{\ell}, \beta L S J J\right)$.

To obtain a similar relation for the nondiagonal elements $\left(S_{0}\left(\beta L S J J^{\prime}\right)\right.$ with $\left.J \neq J^{\prime}\right)$, which represent the relaxation rate of $J$-state interference due to inelastic and superelastic collisions, we make the assumption, also in this case, that the interaction between the atoms and colliders is described by a dipolar operator, and we proceed by analogy with the multiterm atom radiative relaxation rates due to absorption $\left(\mathbb{R}_{A}\right)$ and stimulated emission $\left(\mathbb{R}_{S}\right)$ processes (see Eqs. (7.46a) and (7.46c) of LL04). Assuming an isotropic incident radiation field (i.e. setting $K_{r}=0$ ), such radiative rates assume the simple form

$$
\begin{aligned}
& \mathbb{R}_{\mathbb{A}}\left(\beta L S K Q J J^{\prime} K Q J J^{\prime}\right)=\sum_{\beta_{u} L_{u}} B\left(\beta L S \rightarrow \beta_{u} L_{u} S\right) J_{0}^{0}, \\
& \mathbb{R}_{\mathbb{S}}\left(\beta L S K Q J J^{\prime} K Q J J^{\prime}\right)=\sum_{\beta_{\ell} L_{\ell}} B\left(\beta L S \rightarrow \beta_{\ell} L_{\ell} S\right) J_{0}^{0} .
\end{aligned}
$$

Introducing the inelastic and superelastic collisional rates for transitions between different terms (see Eqs. (27) and (38)), we have

$$
\begin{aligned}
S_{0}\left(\beta L S J J^{\prime}\right) & =\sum_{\beta_{u} L_{u}} C_{I}\left(\beta L S \rightarrow \beta_{u} L_{u} S\right)+\sum_{\beta_{\ell} L_{\ell}} C_{S}\left(\beta L S \rightarrow \beta_{\ell} L_{\ell} S\right) \\
& =\sum_{\beta_{u} L_{u} J_{u}} C_{I}\left(\beta L S J \rightarrow \beta_{u} L_{u} S J_{u}\right)+\sum_{\beta_{\ell} L_{\ell} J_{\ell}} C_{S}\left(\beta L S J \rightarrow \beta_{\ell} L_{\ell} S J_{\ell}\right)=S_{0}(\beta L S J J)=S_{0}(\beta L S) .
\end{aligned}
$$

The relaxation rate of $J$-state interference due to inelastic and superelastic collisions thus coincides with the relaxation rate of $J$-level populations and of interference between magnetic sublevels pertaining to the same $J$-level. This rate, on the other hand, does not depend on the quantum number $J$, and is thus identical for all the $J$-levels of a given term.

When collected together transfer and relaxation rates, the statistical equilibrium equations for the spherical statistical tensors can be written in the form

$$
\begin{aligned}
\frac{\mathrm{d}}{\mathrm{d} t} \beta L S \rho_{Q}^{K}\left(J, J^{\prime}\right)= & \sum_{\beta_{\ell} L_{\ell} J_{\ell} J_{\ell}} \sqrt{\frac{J_{\ell}+J_{\ell}^{\prime}+1}{J+J^{\prime}+1}} C_{I}^{(K)}\left(\beta L S J J^{\prime}, \beta_{\ell} L_{\ell} S J_{\ell} J_{\ell}^{\prime}\right){ }^{\beta_{\ell} L_{\ell} S} \rho_{Q}^{K}\left(J_{\ell}, J_{\ell}^{\prime}\right) \\
& +\sum_{\beta_{u} L_{u} J_{u} J_{u}^{\prime}} \sqrt{\frac{J_{u}+J_{u}^{\prime}+1}{J+J^{\prime}+1}} C_{S}^{(K)}\left(\beta L S J J^{\prime}, \beta_{u} L_{u} S J_{u} J_{u}^{\prime}\right){ }^{\beta_{u} L_{u} S} \rho_{Q}^{K}\left(J_{u}, J_{u}^{\prime}\right) \\
& -S_{0}\left(\beta L S J J^{\prime}\right){ }^{\beta L S} \rho_{Q}^{K}\left(J, J^{\prime}\right) .
\end{aligned}
$$

\section{Application to the case of a two-term atom with unpolarized lower term}

We consider a two-term atom and denote the quantum numbers characterizing the lower and upper term by $\left(\beta_{\ell} L_{\ell} S\right)$ and $\left(\beta_{u} L_{u} S\right)$, respectively. The time evolution of the spherical statistical tensors of the upper term, when taking both radiative (see Eq. (10.115) of LL04) and collisional (inelastic and superelastic collisions only) processes into account is described by the equation

$$
\begin{aligned}
\frac{\mathrm{d}}{\mathrm{d} t} \beta_{u} L_{u} S \rho_{Q}^{K}\left(J_{u}, J_{u}^{\prime}\right)= & -2 \pi \mathrm{i} \sum_{K^{\prime} Q^{\prime} J_{u}^{\prime \prime} J_{u}^{\prime \prime \prime}} N_{\beta_{u} L_{u} S}\left(K Q J_{u} J_{u}^{\prime}, K^{\prime} Q^{\prime} J_{u}^{\prime \prime} J_{u}^{\prime \prime \prime}\right) \beta_{u} L_{u} S \rho_{Q^{\prime}}^{K^{\prime}}\left(J_{u}^{\prime \prime}, J_{u}^{\prime \prime \prime}\right) \\
& +\sum_{K^{\prime} Q^{\prime} J_{\ell} J_{\ell}^{\prime}} \mathbb{T}_{A}\left(\beta_{u} L_{u} S K Q J_{u} J_{u}^{\prime}, \beta_{\ell} L_{\ell} S K^{\prime} Q^{\prime} J_{\ell} J_{\ell}^{\prime}\right) \beta_{\ell} L_{\ell} S \rho_{Q^{\prime}}^{K^{\prime}}\left(J_{\ell}, J_{\ell}^{\prime}\right) \\
& -\sum_{K^{\prime} Q^{\prime} J_{u}^{\prime \prime} J_{u}^{\prime \prime \prime}}\left[\mathbb{R}_{E}\left(\beta_{u} L_{u} S K Q J_{u} J_{u}^{\prime} K^{\prime} Q^{\prime} J_{u}^{\prime \prime} J_{u}^{\prime \prime \prime}\right)\right. \\
& \left.+\mathbb{R}_{S}\left(\beta_{u} L_{u} S K Q J_{u} J_{u}^{\prime} K^{\prime} Q^{\prime} J_{u}^{\prime \prime} J_{u}^{\prime \prime \prime}\right)\right] \beta_{u} L_{u} S \rho_{Q^{\prime}}^{K^{\prime}}\left(J_{u}^{\prime \prime}, J_{u}^{\prime \prime \prime}\right) \\
& +\sum_{J_{\ell} J_{\ell}^{\prime}} \sqrt{\frac{J_{\ell}+J_{\ell}^{\prime}+1}{J_{u}+J_{u}^{\prime}+1}} C_{I}^{(K)}\left(\beta_{u} L_{u} S J_{u} J_{u}^{\prime}, \beta_{\ell} L_{\ell} S J_{\ell} J_{\ell}^{\prime}\right) \beta_{\ell} L_{\ell} S \rho_{Q}^{K}\left(J_{\ell}, J_{\ell}^{\prime}\right) \\
& -S_{0}\left(\beta_{u} L_{u} S J_{u} J_{u}^{\prime}\right) \beta_{u} L_{u} S \rho_{Q}^{K}\left(J_{u}, J_{u}^{\prime}\right),
\end{aligned}
$$

where $N$ is the magnetic kernel (see Eq. (7.41) of LL04), $\mathbb{T}_{A}$ the radiative transfer rate due to absorption, while $\mathbb{R}_{E}$ and $\mathbb{R}_{S}$ are the radiative relaxation rates due to spontaneous and stimulated emission, respectively. 
We now make the following simplifying assumptions:

- There is no magnetic field. Under this assumption the kernel $N$ takes the simpler form

$N_{\beta_{u} L_{u} S}\left(K Q J_{u} J_{u}^{\prime}, K^{\prime} Q^{\prime} J_{u}^{\prime \prime} J_{u}^{\prime \prime \prime}\right)=\delta_{K K^{\prime}} \delta_{Q Q^{\prime}} \delta_{J_{u} J_{u}^{\prime \prime}} \delta_{J_{u}^{\prime} J_{u}^{\prime \prime \prime}} v_{\beta_{u} L_{u} S J_{u}, \beta_{u} L_{u} S J_{u}^{\prime}}$,

with $v_{\beta_{u} L_{u} S J_{u}, \beta_{u} L_{u} S J_{u}^{\prime}}=\left[E\left(\beta_{u} L_{u} S J_{u}\right)-E\left(\beta_{u} L_{u} S J_{u}^{\prime}\right)\right] / h$, where $E(\beta L S J)$ is the energy of a given fine-structure $J$-level, and $h$ is the Planck constant.

- The radiation field is weak so that stimulated emission can be neglected $\left(\mathbb{R}_{S}=0\right)$.

- The lower term is unpolarized (i.e., the magnetic sublevels of the lower term are evenly populated and no interference is present between them). Under this assumption the spherical statistical tensors of the lower term are given by

$$
\beta_{\ell} L_{\ell} S \rho_{Q}^{K}\left(J_{\ell}, J_{\ell}^{\prime}\right)=\delta_{K 0} \delta_{Q 0} \delta_{J_{\ell} J_{\ell}^{\prime}} \frac{\sqrt{2 J_{\ell}+1}}{(2 S+1)\left(2 L_{\ell}+1\right)} \frac{\mathbb{N}_{\ell}}{\mathcal{N}}
$$

where $\mathcal{N}$ is total number density of atoms, and $\mathbb{N}_{\ell}$ the number density of atoms in the lower term.

- The electron-atom interaction is described by a dipolar operator. Under this assumption, defining through Eq. (38) a superelastic collisional rate for the transition from the upper to the lower term $\left(C_{S}\left(\beta_{u} L_{u} S \rightarrow \beta_{\ell} L_{\ell} S\right)\right)$, the collisional relaxation rate is given by (see Eq. (53))

$$
S_{0}\left(\beta_{u} L_{u} S J_{u} J_{u}^{\prime}\right)=C_{S}\left(\beta_{u} L_{u} S \rightarrow \beta_{\ell} L_{\ell} S\right) .
$$

Taking the above-mentioned assumptions into account, and recalling that (see Eq. (7.46b) of LL04) $\mathbb{R}_{E}\left(\beta_{u} L_{u} S K Q J_{u} J_{u}^{\prime} K^{\prime} Q^{\prime} J_{u}^{\prime \prime} J_{u}^{\prime \prime \prime}\right)=$ $\delta_{K K^{\prime}} \delta_{Q Q^{\prime}} \delta_{J_{u} J_{u}^{\prime \prime}} \delta_{J_{u}^{\prime} J_{u}^{\prime \prime \prime}} A\left(\beta_{u} L_{u} S \rightarrow \beta_{\ell} L_{\ell} S\right)$, we obtain

$$
\begin{aligned}
\frac{\mathrm{d}}{\mathrm{d} t} \beta_{u} L_{u} S \rho_{Q}^{K}\left(J_{u}, J_{u}^{\prime}\right)= & -2 \pi \mathrm{i}{\gamma_{\beta_{u}} L_{u} S J_{u}, \beta_{u} L_{u} S J_{u}^{\prime}}^{\beta_{u} L_{u} S} \rho_{Q}^{K}\left(J_{u}, J_{u}^{\prime}\right) \\
& +\sum_{J_{\ell}} \mathbb{T}_{A}\left(\beta_{u} L_{u} S K Q J_{u} J_{u}^{\prime}, \beta_{\ell} L_{\ell} S 00 J_{\ell} J_{\ell}\right) \frac{\sqrt{2 J_{\ell}+1}}{(2 S+1)\left(2 L_{\ell}+1\right)} \frac{\mathbb{N}_{\ell}}{\mathcal{N}} \\
& -A\left(\beta_{u} L_{u} S \rightarrow \beta_{\ell} L_{\ell} S\right){ }^{\beta_{u} L_{u} S} \rho_{Q}^{K}\left(J_{u}, J_{u}^{\prime}\right) \\
& +\delta_{K 0} \delta_{Q 0} \sum_{J_{\ell}} \sqrt{\frac{2 J_{\ell}+1}{2 J_{u}+1}} C_{I}^{(0)}\left(\beta_{u} L_{u} S J_{u} J_{u}, \beta_{\ell} L_{\ell} S J_{\ell} J_{\ell}\right) \frac{\sqrt{2 J_{\ell}+1}}{(2 S+1)\left(2 L_{\ell}+1\right)} \frac{\mathbb{N}_{\ell}}{\mathcal{N}} \\
& -C_{S}\left(\beta_{u} L_{u} S \rightarrow \beta_{\ell} L_{\ell} S\right) \beta_{u} L_{u} S \rho_{Q}^{K}\left(J_{u}, J_{u}^{\prime}\right) .
\end{aligned}
$$

As expected, under the hypotheses of isotropic collisions and unpolarized lower term, transfer processes due to inelastic collisions only contribute to the time evolution of the 0-rank spherical statistical tensors of the upper term. Assuming that the colliding particles are characterized by a Maxwellian velocity distribution, the collisional rates $C_{S}^{(0)}$ and $C_{I}^{(0)}$ can be related through the Milne-Einstein relation

$C_{S}^{(0)}\left(\beta_{\ell} L_{\ell} S J_{\ell} J_{\ell}, \beta_{u} L_{u} S J_{u} J_{u}\right)=\frac{2 J_{\ell}+1}{2 J_{u}+1} C_{I}^{(0)}\left(\beta_{u} L_{u} S J_{u} J_{u}, \beta_{\ell} L_{\ell} S J_{\ell} J_{\ell}\right) \exp \left[\frac{E\left(\beta_{u} L_{u} S J_{u}\right)-E\left(\beta_{\ell} L_{\ell} S J_{\ell}\right)}{K_{B} T}\right]$,

where $T$ is the electron temperature. Using Eq. (38), the fourth term on the righthand side of Eq. (59) can be written in the form

$\delta_{K 0} \delta_{Q 0} C_{S}\left(\beta_{u} L_{u} S \rightarrow \beta_{\ell} L_{\ell} S\right) \frac{c^{2}}{2 h v_{0}^{3}} B_{T}\left(v_{0}\right) \frac{\sqrt{2 J_{u}+1}}{(2 S+1)\left(2 L_{\ell}+1\right)} \frac{\mathbb{N}_{\ell}}{\mathcal{N}}$,

where $B_{T}\left(v_{0}\right)$ is the Planck function in the Wien limit (consistently with the assumption of neglecting stimulated emission) at temperature $T$, and where $v_{0}$ is the Bohr frequency corresponding to the energy difference between the centers of gravity of the two terms. (We neglect the frequency differences among the various components of the multiplet in the exponential appearing in the Milne-Einstein relation.)

Taking the expression of $\mathbb{T}_{A}\left(\beta_{u} L_{u} S K Q J_{u} J_{u}^{\prime}, \beta_{\ell} L_{\ell} S 00 J_{\ell} J_{\ell}\right)$ (see Eq. (10.124) of LL04) into account and performing the sum over $J_{\ell}$ using Eq. (A.6), the second term on the righthand side of Eq. (59) is given by

$B\left(\beta_{\ell} L_{\ell} S \rightarrow \beta_{u} L_{u} S\right)(-1)^{1-L_{\ell}+S+J_{u}^{\prime}+K+Q}\left\{\begin{array}{ccc}1 & 1 & K \\ L_{u} & L_{u} & L_{\ell}\end{array}\right\}\left\{\begin{array}{ccc}L_{u} & L_{u} & K \\ J_{u} & J_{u}^{\prime} & S\end{array}\right\} J_{-Q}^{K}\left(v_{0}\right) \frac{\sqrt{3\left(2 J_{u}+1\right)\left(2 J_{u}^{\prime}+1\right)}}{2 S+1} \frac{\mathbb{N}_{\ell}}{\mathcal{N}}$

We recall that the quantum theory of polarization described in LL04 is valid under the so-called flat spectrum approximation (that is, the incident radiation field that produces optical pumping in the atomic system must be flat over a frequency interval $\Delta v$ larger than the natural width of the atomic levels, and, when coherence between nondegenerate levels is involved, $\Delta v$ must then be larger than the corresponding Bohr frequency). For this reason, it is sufficient to evaluate the radiation field tensor $J_{Q}^{K}$ (see Eq. (5.157) of LL04 for its definition) at a single frequency within the multiplet. 
In stationary situations, recalling the relations among the Einstein coefficients

$B\left(\beta_{\ell} L_{\ell} S \rightarrow \beta_{u} L_{u} S\right)=\frac{2 L_{u}+1}{2 L_{\ell}+1} B\left(\beta_{u} L_{u} S \rightarrow \beta_{\ell} L_{\ell} S\right)=\frac{2 L_{u}+1}{2 L_{\ell}+1} \frac{c^{2}}{2 h v_{0}^{3}} A\left(\beta_{u} L_{u} S \rightarrow \beta_{\ell} L_{\ell} S\right)$,

the spherical statistical tensors of the upper term are given by

$$
\begin{aligned}
\beta_{u} L_{u} S \rho_{Q}^{K}\left(J_{u}, J_{u}^{\prime}\right)= & \frac{\sqrt{2 J_{u}+1}}{(2 S+1)\left(2 L_{u}+1\right)} \frac{\mathbb{N}_{\ell}}{\mathcal{N}} \frac{B\left(\beta_{\ell} L_{\ell} S \rightarrow \beta_{u} L_{u} S\right)}{A\left(\beta_{u} L_{u} S \rightarrow \beta_{\ell} L_{\ell} S\right)} \\
& \times \frac{\left(2 L_{u}+1\right) \sqrt{3\left(2 J_{u}^{\prime}+1\right)}(-1)^{1-L_{\ell}+S+J_{u}^{\prime}+K+Q}\left\{\begin{array}{ccc}
1 & 1 & K \\
L_{u} & L_{u} & L_{\ell}
\end{array}\right\}\left\{\begin{array}{ccc}
L_{u} & L_{u} & K \\
J_{u} & J_{u}^{\prime} & S
\end{array}\right\} J_{-Q}^{K}\left(v_{0}\right)+\epsilon^{\prime} B_{T}\left(v_{0}\right) \delta_{K 0} \delta_{Q 0}}{1+\epsilon^{\prime}+2 \pi i v_{\beta_{u} L_{u} S J_{u}, \beta_{u} L_{u} S J_{u}^{\prime}} / A\left(\beta_{u} L_{u} S \rightarrow \beta_{\ell} L_{\ell} S\right)},
\end{aligned}
$$

where, in analogy with the two-level atom case, we have introduced the quantity

$\epsilon^{\prime}=\frac{C_{S}\left(\beta_{u} L_{u} S \rightarrow \beta_{\ell} L_{\ell} S\right)}{A\left(\beta_{u} L_{u} S \rightarrow \beta_{\ell} L_{\ell} S\right)}$

It can be easily proven that if $S=0$, so that the upper and lower terms are composed by a single fine-structure $J$-level, the expression of a two-level atom is recovered (see Eq. (10.50) of LL04, with $H_{u}=\delta_{u}^{(K)}=0$ ).

Substituting Eq. (64) into Eq. (10.127) of LL04, and introducing the frequency-integrated absorption coefficient of the multiplet

$k_{M}^{A}=\frac{h v_{0}}{4 \pi} \mathbb{N}_{\ell} B\left(\beta_{\ell} L_{\ell} S \rightarrow \beta_{u} L_{u} S\right)$,

and the absorption profile of the multiplet (in the absence of magnetic fields, and for the case of a two-term atom with unpolarized lower term)

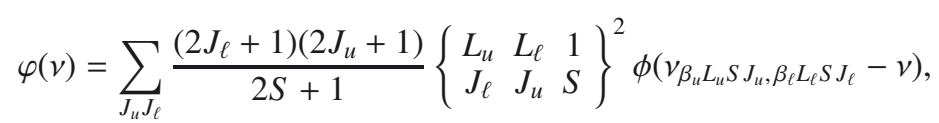

where $\phi\left(v_{0}-v\right)$ are Lorentzian profiles centered at the frequencies of the various components of the multiplet, we find the following expression of the emission coefficient in the four Stokes parameters:

$$
\begin{aligned}
& \varepsilon_{i}(\nu, \boldsymbol{\Omega})=k_{M}^{A} \frac{2 L_{u}+1}{2 S+1} \sum_{K Q} \sum_{J_{u} J_{u}^{\prime} J_{\ell}}(-1)^{S-L_{\ell}+J_{u}+J_{u}^{\prime}+J_{\ell}+K+Q} 3\left(2 J_{u}+1\right)\left(2 J_{u}^{\prime}+1\right)\left(2 J_{\ell}+1\right) \\
& \times\left\{\begin{array}{ccc}
L_{u} & L_{\ell} & 1 \\
J_{\ell} & J_{u} & S
\end{array}\right\}\left\{\begin{array}{lll}
L_{u} & L_{\ell} & 1 \\
J_{\ell} & J_{u}^{\prime} & S
\end{array}\right\}\left\{\begin{array}{ccc}
1 & 1 & K \\
J_{u} & J_{u}^{\prime} & J_{\ell}
\end{array}\right\}\left\{\begin{array}{ccc}
1 & 1 & K \\
L_{u} & L_{u} & L_{\ell}
\end{array}\right\}\left\{\begin{array}{ccc}
L_{u} & L_{u} & K \\
J_{u} & J_{u}^{\prime} & S
\end{array}\right\} \\
& \times \mathcal{T}_{Q}^{K}(i, \boldsymbol{\Omega}) J_{-Q}^{K}\left(v_{0}\right) \frac{1}{2} \frac{\Phi\left(v_{\beta_{u} L_{u} S J_{u}, \beta_{\ell} L_{\ell} S J_{\ell}}-v\right)+\Phi\left(v_{\beta_{u} L_{u} S J_{u}^{\prime}, \beta_{\ell} L_{\ell} S J_{\ell}}-v\right)^{*}}{1+\epsilon^{\prime}+2 \pi \mathrm{i} v_{\beta_{u} L_{u} S J_{u}^{\prime}, \beta_{u} L_{u} S J_{u}} / A\left(\beta_{u} L_{u} S \rightarrow \beta_{\ell} L_{\ell} S\right)} \\
& +\frac{\epsilon^{\prime}}{1+\epsilon^{\prime}} k_{M}^{A} B_{T}\left(v_{0}\right) \varphi(v) \delta_{i, 0},
\end{aligned}
$$

with $i=0,1,2$, and 3, standing for Stokes $I, Q, U$ and $V$, respectively. Here, $v$ and $\Omega$ are the frequency and propagation direction of the emitted radiation, respectively, $\mathcal{T}_{Q}^{K}(i, \boldsymbol{\Omega})$ is the geometrical tensor introduced by Landi Degl'Innocenti $(1983)$, and $\Phi\left(v_{0}-v\right)$ is the complex emission profile

$\Phi\left(v_{0}-v\right)=\phi\left(v_{0}-v\right)+\mathrm{i} \psi\left(v_{0}-v\right)$,

with $\phi\left(v_{0}-v\right)$ a Lorentzian profile and $\psi\left(v_{0}-v\right)$ the associated dispersion profile ${ }^{5}$. The last term on the righthand side of Eq. (68) represents the contribution to the emission coefficient coming from collisionally excited atoms. Since collisions are assumed isotropic, this term only contributes to Stokes- $I$.

As an example suitable to illustrating the sensitivity of the emergent scattering line polarization to the studied collisional rates, we consider an isothermal slab of stellar atmospheric plasma located at a given height above the surface of a solar-like star and characterized by a given optical depth $\Delta \tau$. Neglecting limb-darkening effects, the radiation illuminating the slab from below is characterized by an anisotropy factor $w=\sqrt{2} J_{0}^{2} / J_{0}^{0}$ given by

$w=\frac{\cos \alpha(1+\cos \alpha)}{2}$

\footnotetext{
5 The equations derived here are valid in the atom rest frame. Nevertheless, under the assumption of complete redistribution on velocities (see Chapter 13 of LL04), the same equations can also be applied in the observer's frame, with $\phi\left(v_{0}-v\right)$ and $\psi\left(v_{0}-v\right)$ the Voigt profile and the Faraday-Voigt profile, respectively (provided that the atoms have a Maxwellian distribution of velocities).
} 


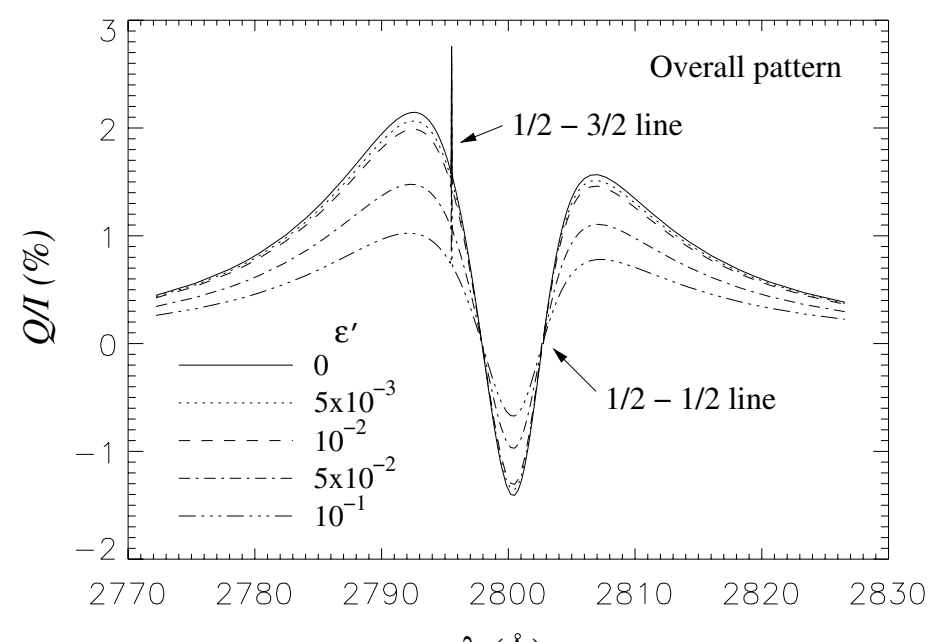

$\lambda(\AA)$

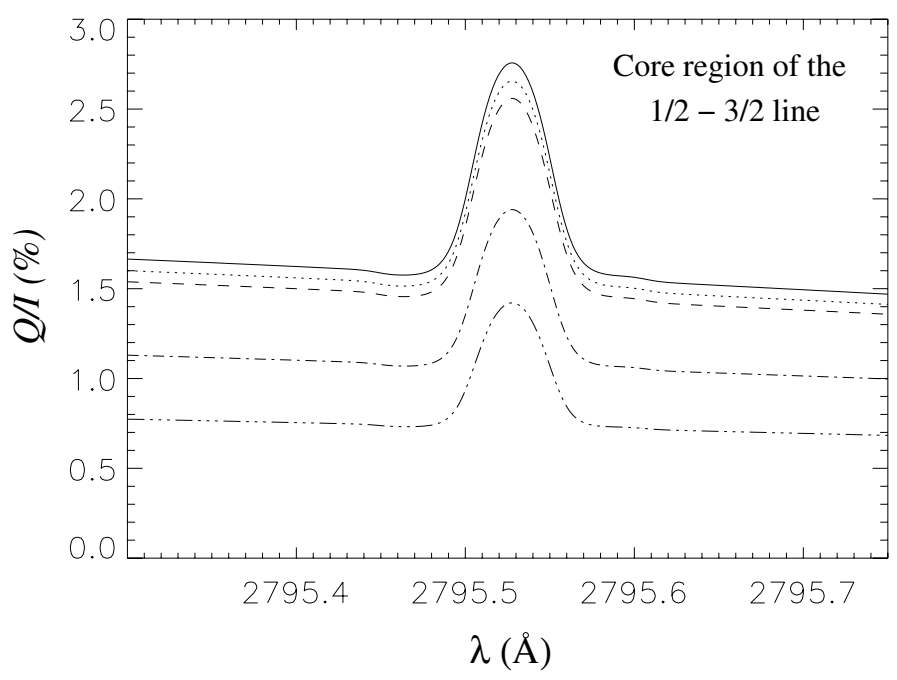

$\lambda(\AA)$

Fig. 1. Left panel: $Q / I$ profile of the radiation emitted across a ${ }^{2} \mathrm{~S}-{ }^{2} \mathrm{P}$ transition (the level energies and transition probabilities are those of the $\mathrm{Mg}$ II $h$ and $k$ lines) as obtained for different values of the parameter $\epsilon^{\prime}$ (indicated in the plot). The arrows point to the wavelength positions of the two lines. Right panel: zoom of the line-core region of the $1 / 2-3 / 2$ transition. We consider the radiation emitted at $\mu=0.1$ by a slab located 0.03 stellar radii above the surface, and with an optical depth (at the line-center frequency of the $1 / 2-1 / 2$ transition) $\Delta \tau=0.5$. We solve the full nonLTE radiative transfer problem within the slab, the boundary condition being the stellar radiation illuminating the slab from below (limb-darkening effects are neglected). We consider a Doppler width of $26 \mathrm{~m} \AA$, corresponding to a temperature of $10^{4} \mathrm{~K}$ and a microturbulent velocity of $1 \mathrm{~km} \mathrm{~s}^{-1}$. We include the effect of an unpolarized continuum characterized by an opacity $10^{8}$ times less than the line opacity at the line-center frequency of the $1 / 2-1 / 2$ transition. The reference direction for positive $Q$ is the parallel to the closest limb.

where $\alpha$ is half the angle subtended by the stellar disk, as seen from the slab. We solve the equations of the non-LTE problem described in this section for the case of a ${ }^{2} \mathrm{~S}-{ }^{2} \mathrm{P}$ transition, using the level energies and transition probabilities of the Mg II $h$ and $k$ lines, and we calculate the fractional linear polarization of the radiation emerging at $\mu=\cos \theta=0.1$, with $\theta$ the angle formed by the local vertical (perpendicular to the slab) and the emission direction. The non-LTE radiative transfer problem is solved following the numerical methods described in Trujillo Bueno \& Manso Sainz (1999).

Figure 1 shows the fractional linear polarization $Q / I$ pattern calculated for different values of $\epsilon^{\prime}$, considering a slab located 0.03 stellar radii above the surface (corresponding to about $2 \times 10^{4} \mathrm{~km}$ in the solar case), and characterized by an optical depth (at the line-center frequency of the $1 / 2-1 / 2$ transition) $\Delta \tau=0.5$. We assume a Doppler width of $26 \mathrm{~m} \AA$, corresponding to a temperature of $10^{4} \mathrm{~K}$, and a microturbulent velocity of $1 \mathrm{~km} \mathrm{~s}^{-1}$. The damping constant is consistently calculated as

$a=\frac{\Gamma}{\Delta v_{D}}=\frac{\gamma_{u}}{4 \pi \Delta v_{D}}$

with $\gamma_{u}=A\left(\beta_{u} L_{u} S \rightarrow \beta_{\ell} L_{\ell} S\right)+C_{S}\left(\beta_{u} L_{u} S \rightarrow \beta_{\ell} L_{\ell} S\right)$ the inverse lifetime of the upper term (elastic collisions, hence their broadening effect, are neglected). We include the contribution of an unpolarized continuum characterized by an opacity $\eta_{I}^{c}=10^{-8} \eta_{I}^{\ell}\left(v_{1 / 2-1 / 2}\right)$, with $\eta_{I}^{\ell}\left(v_{1 / 2-1 / 2}\right)=k_{M}^{A} \varphi\left(v_{1 / 2-1 / 2}\right)$ the line opacity at the line-center frequency of the $1 / 2-1 / 2$ transition. We first note that in the slab model that we have considered (in which radiative transfer effects are significant), the $Q / I$ profiles show the typical signatures of $J$-state interference, such as the sign-reversal between the two lines, and the high polarization values in the far wings (see Stenflo 1980, LL04; Belluzzi \& Trujillo Bueno 2011). As can be observed, the modification of the scattering line polarization pattern due to inelastic and superelastic collisions (quenching effect) becomes appreciable only for rather high values of the collisional rates $\left(\epsilon^{\prime} \gtrsim 10^{-2}\right)$.

\section{Conclusions}

In this paper we have formally defined and investigated the transfer and relaxation rates due to isotropic inelastic and superelastic collisions that enter the statistical equilibrium equations for the atomic density matrix of a multiterm atom (i.e., a model atom accounting for quantum interference between magnetic sublevels pertaining either to the same $J$-level, or to different $J$-levels within the same term).

While the numerical values of the collisional rates for $J$-level populations are generally available (either from approximate theoretical expressions or from experimental data), the values of the collisional rates describing the transfer and relaxation of quantum coherence are in most cases unknown. In this work we focused our attention on the collisional rates for $J$-state interference (the physical aspect that cannot be accounted for with a multilevel model atom). Under the assumption that the interaction between the atom and the perturber is described by a dipolar operator, we derived suitable relations between such rates and the usual collisional rates for $J$-level populations. In particular, we showed that the collisional relaxation rate for $J$-state interference coincide 
with the relaxation rate for $J$-level populations and for interference between magnetic sublevels pertaining to the same $J$-level. We also observed that this rate does not depend on the particular $J$-level under consideration, so that it is sufficient to introduce a single collisional relaxation rate for the whole term. As a consistency proof of our derivations, we showed that the transfer and relaxation rates for $J$-level populations and for interference between pairs of magnetic sublevels pertaining to the same $J$-level reduce to those derived in Sect. 7.13 of LL04 for the multilevel atom case.

As an illustrative application, we considered an isothermal slab of given optical depth, located at a given height above the surface of a solar-like star, and anisotropically illuminated by its photospheric radiation field. The numerical solution of the full non-LTE problem for the case of a two-term atom with unpolarized lower term shows that the polarization of the radiation emerging from the slab at $\mu=0.1$ is sensitive to the presence of isotropic inelastic and superelastic collisions only for values of the parameter $\epsilon^{\prime}=C_{S}\left(\beta_{u} L_{u} S \rightarrow \beta_{\ell} L_{\ell} S\right) / A\left(\beta_{u} L_{u} S \rightarrow \beta_{\ell} L_{\ell} S\right)$ on the order of $10^{-2}$ or larger. Such values are actually needed to produce an appreciable variation in the $Q / I$ profiles of the emergent radiation.

Acknowledgements. Financial support by the Spanish Ministry of Science through projects AYA2010-18029 (Solar Magnetism and Astrophysical Spectropolarimetry) and CONSOLIDER INGENIO CSD2009-00038 (Molecular Astrophysics: The Herschel and Alma Era) is gratefully acknowledged.

\section{Appendix A: Properties of 3-j and 6-j symbols and of rotation matrices}

In this appendix we recall some useful properties and relations of 3- $j$ and $6-j$ symbols, as well as of rotation matrices that are used in the derivation of the expressions presented in the paper. A proof of these properties can be found in Chap. 2 of LL04.

- Symmetry properties of $3-j$ symbols:

The $3-j$ symbols are invariant under cyclic permutations of their columns and are multiplied by $(-1)^{a+b+c}$ under noncyclic ones

$\left(\begin{array}{ccc}a & b & c \\ \alpha & \beta & \gamma\end{array}\right)=\left(\begin{array}{lll}b & c & a \\ \beta & \gamma & \alpha\end{array}\right)=(-1)^{a+b+c}\left(\begin{array}{ccc}c & b & a \\ \gamma & \beta & \alpha\end{array}\right)$, etc.

The $3-j$ symbols are multiplied by $(-1)^{a+b+c}$ under sign inversion of the second row

$$
\left(\begin{array}{ccc}
a & b & c \\
\alpha & \beta & \gamma
\end{array}\right)=(-1)^{a+b+c}\left(\begin{array}{ccc}
a & b & c \\
-\alpha & -\beta & -\gamma
\end{array}\right) \text {. }
$$

- Orthogonality relation of $3-j$ symbols

$$
\sum_{\alpha \beta}(2 c+1)\left(\begin{array}{ccc}
a & b & c \\
\alpha & \beta & \gamma
\end{array}\right)\left(\begin{array}{ccc}
a & b & c^{\prime} \\
\alpha & \beta & \gamma^{\prime}
\end{array}\right)=\delta_{c c^{\prime}} \delta_{\gamma \gamma^{\prime}}
$$

- Analytical expression of 3-j symbols for particular values of the arguments:

$$
\left(\begin{array}{ccc}
a & b & 0 \\
\alpha & \beta & 0
\end{array}\right)=(-1)^{a-\alpha} \delta_{a b} \delta_{\alpha,-\beta} \frac{1}{\sqrt{2 a+1}} .
$$

- Symmetry properties of $6-j$ symbols:

The 6- $j$ symbols are invariant under interchange of any two columns and under interchange of the upper and lower arguments in any two columns.

- Sum rules of 6- $j$ symbols:

$$
\begin{aligned}
& \sum_{c}(2 c+1)(2 f+1)\left\{\begin{array}{lll}
a & b & c \\
d & e & f
\end{array}\right\}\left\{\begin{array}{lll}
a & b & c \\
d & e & g
\end{array}\right\}=\delta_{f g}, \\
& \sum_{c}(-1)^{a+b+c+d+e+f+g+h+i+j}(2 c+1)\left\{\begin{array}{lll}
a & b & c \\
d & e & f
\end{array}\right\}\left\{\begin{array}{lll}
a & b & c \\
g & h & i
\end{array}\right\}\left\{\begin{array}{lll}
g & h & c \\
e & d & j
\end{array}\right\}=\left\{\begin{array}{lll}
f & i & j \\
g & d & b
\end{array}\right\}\left\{\begin{array}{lll}
f & i & j \\
h & e & a
\end{array}\right\} .
\end{aligned}
$$

- Analytical expression of 6- $j$ symbols for particular values of the arguments

$$
\left\{\begin{array}{lll}
a & b & 0 \\
d & e & f
\end{array}\right\}=\delta_{a b} \delta_{e d}(-1)^{a+e+f} \frac{1}{\sqrt{(2 a+1)(2 d+1)}} .
$$

- Contraction of 3-j symbols:

$$
\sum_{f}(-1)^{a+b+c+d-e+f-\alpha-\delta}(2 f+1)\left\{\begin{array}{lll}
a & b & e \\
d & c & f
\end{array}\right\}\left(\begin{array}{lll}
c & a & f \\
\gamma & \alpha & \phi
\end{array}\right)\left(\begin{array}{ccc}
b & d & f \\
\beta & \delta & -\phi
\end{array}\right)=\left(\begin{array}{ccc}
a & b & e \\
\alpha & \beta & -\epsilon
\end{array}\right)\left(\begin{array}{lll}
d & c & e \\
\delta & \gamma & \epsilon
\end{array}\right) .
$$


- Orthogonality relations of rotation matrices

$\sum_{P} \mathcal{D}_{P N}^{J}(R)^{*} \mathcal{D}_{P M}^{J}(R)=\delta_{M N}$

- Product of two rotation matrices:

$$
\begin{aligned}
& \mathcal{D}_{M N}^{J}(R) \mathcal{D}_{M^{\prime} N^{\prime}}^{J^{\prime}}(R)^{*}=(-1)^{M^{\prime}-N^{\prime}} \sum_{K}(2 K+1)\left(\begin{array}{ccc}
J & J^{\prime} & K \\
M & -M^{\prime} & Q
\end{array}\right)\left(\begin{array}{ccc}
J & J^{\prime} & K \\
N & -N^{\prime} & Q^{\prime}
\end{array}\right) \mathcal{D}_{Q Q^{\prime}}^{K}(R)^{*}, \\
& \mathcal{D}_{M N}^{J}(R) \mathcal{D}_{M^{\prime} N^{\prime}}^{J^{\prime}}(R)=\sum_{K}(2 K+1)\left(\begin{array}{ccc}
J & J^{\prime} & K \\
M & M^{\prime} & Q
\end{array}\right)\left(\begin{array}{ccc}
J & J^{\prime} & K \\
N & N^{\prime} & Q^{\prime}
\end{array}\right) \mathcal{D}_{Q Q^{\prime}}^{K}(R)^{*} .
\end{aligned}
$$

\section{References}

Belluzzi, L., \& Trujillo Bueno, J. 2011, ApJ, 743, 3

Bommier, V. 2009, in Solar Polarization 5, eds. S.V. Berdyugina, K. N. Nagendra, \& R. Ramelli, ASP Conf. Ser., 405, 335 Landi Degl'Innocenti, E. 1983, Sol. Phys., 85, 1

Landi Degl'Innocenti, E., \& Landolfi, M. 2004, Polarization in Spectral Lines (Dordrecht: Kluwer)

Seaton, M. J. 1962, in Atomic and Molecular Processes, ed. D.R. Bates (New York: Academic Press)

Stenflo, J. O. 1980, A\&A, 84, 68

Trujillo Bueno, J., \& Manso Sainz, R. 1999, ApJ, 516, 436

Van Regemorter, H. 1962, ApJ, 136, 906 